\title{
Journal of Stem Cells \& Regenerative Medicine
}

An exclusive \& FREE online journal

www.pubstemcell.com

JSRM/Vol5 No.1, 2009; p1-2

\section{Editorial}

\section{Expanding horizons}

\section{Dear friends,}

The debate of whether Stem Cell Therapy is a hype or hope has been raging for quite some time and has been rekindled in the current year.

Stem Cell Scientists have been particularly enthused by the bold standard taken by Barack Obama in passing an Executive Order that lifted the ban on federal funding of Research on Embryonic Stem Cell (ESC) lines created after August 9, 2001. With the lifting of the ban more money is expected to be poured into ESC and Stem Cell Research in general and this augurs well for this emerging science. This is hope.

Amariglio et al have reported the occurrence of a multifocal brain tumor in a boy with ataxia telengectasia four years after he was treated with intracerebella and intrathecal injection of human fetal neural stem cells. Molecular and Cytogenetic studies showed that the tumor was of non host origin raising the possibility of it being derived from transplanted neural stem cell. This is the first report of a donor-derived brain tumor in neural stem cell therapy and opens a Pandora's Box of questions about the safety of such therapies. This signifies the hype surrounding the therapy.

However controversies are a part of any emerging science. Our goals should be to march forward conducting our research under strict ethical principles and rigorous oversight, ironing out even minor flaws, always being on the lookout for adverse events and identifying ways and means of preventing their occurrence in future.

JSRM has been in receipt of six articles, which speaks well for the interest people have for stem cell science in general and our journal in particular.

The articles we have received for this edition of JSRM cover all aspects necessary for a science. Rosen et al have described the percentage variation of adipose stromal cells isolated from two different inbred mouse strains and Bhonde et al have reported the existence of multipotent stem cells in human fallopian tube. If cells can be identified, isolated they need suitable media for expansion and delivery. Dr. H.N. Madhavan et al article describes the use of such a medium (Mebiol Gel) for transplanting corneal limbal stem cells. Even if cells are ready for delivery, we should first have suitable animal models to test them and the article Garikipati Venkata Naga Srikanth et al is a good article describing are such animal model, that can be used to testing efficacy of stem cell.

Aoyama et al article on using cell therapy for avascular osteonecrosis of femoral head reiterates the fact that stem cell therapy has a potential to cure a wide range of diseases and encourages all of us to continue in our guest to take stem cell therapy to treat hither to untreatable diseases.

The article by Senthil Kumar Pazhanisamy et al highlights the fact that our current understanding of genome instabilities is limited. It is my personal opinion that with a 
better understanding of genome organization (which may take several years) we can possibly predict the occurrence of tumorogenesis and reject such stem cells obtained at source from possible use in therapy. So that we would have no more cases of tumors post stem cell therapy

Yours sincerely,

The Editorial team. 